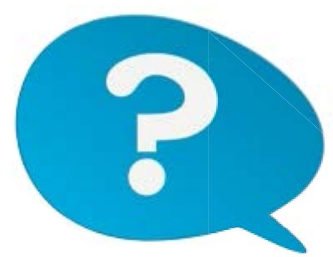

\title{
Ist nach einer Mononukleose eine Tonsillektomie generell anzuraten?
}

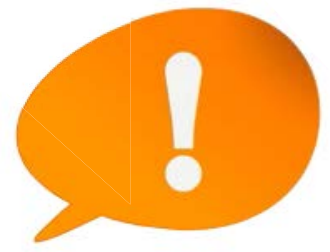

Frage: Wie wird es medizinisch begründet, dass viele HNO-Ärzte nach einer stattgehabten EBV-Infektion (Mononukleose-Tonsillitis) "generell“ nach einem freien Intervall die Durchführung einer Tonsillektomie empfehlen?

Antwort: Die Tonsillektomie ist eine wichtige therapeutische Option, die bezüglich ihrer Indikation klar geregelt ist. Hier hat es in den letzten Jahren kaum nennenswerte Änderungen gegeben. Dennoch erarbeiten bzw. überarbeiten in regelmäßigen Abständen Fachkommissionen Leitlinien zu den Indikationen im Kindesalter und im Erwachsenenalter. Im Gegensatz zur Vorgehens- weise in vergangenen Jahrzehnten, wird die Indikation heute wegen des nicht zu unterschätzenden Risikos der Nachblutung strenger gestellt. Im Kindesalter ist das Nachblutungsrisiko grundsätzlich geringer als im Erwachsenenalter. Tritt eine Nachblutung auf, ist diese im Kindesalter allerdings gefährlicher $[1,2]$.

Es gibt fünf klassische Indikationen zur Tonsillektomie:

- Kontakttonsillen (mechanisches Hindernis für Atmung)

_rezidivierende bakterielle (!) Tonsillitiden, öfter als 5-6 mal pro Jahr

_chronische Tonsillitiden mit dem Risiko eines Fokus

_einseitige Tonsillenvergrößerung

\begin{abstract}
Neue Fortbildungsveranstaltung "consilium HNO live“
„Tonsillektomie, Tonsillotomie oder konservativ - Wann, was, wie bei Tonsillitis?“ Das ist nur eines der Themen, die in einer neuen ganztägigen Fortbildungsveranstaltung am 16. September dieses Jahres behandelt werden, dem "1. consilium HNO live“. Dieses neue Symposium unter wissenschaftlicher Leitung von Prof. Dr. Thomas Deitmer und Dr. Christian Sieling, beide Dortmund, richtet sich speziell an niedergelassene HNO-Ärzte/innen.

Das "consilium HNO live" steht für praxisrelevante Themen, produktneutrale Wissensvermittlung und hochkarätige Experten. Ein Thema wird 40 min behandelt: 20 min Vortrag, $10 \mathrm{~min}$ Falldiskussion und $10 \mathrm{~min}$ für Ihre Fragen an den Referenten. Ein umfassendes Handout, strenges Zeitmanagement und der persönliche Austausch mit den Referenten im Rahmen der "consilium corner" in den Pausen runden das didaktische Konzept ab. Das Ziel: maximaler praktischer Nutzen für den Teilnehmer.
\end{abstract}

\section{Die Daten im Überblick:}

$\begin{array}{ll}\text { Veranstaltung: } & \text { 1. consilium HNO live } \\ \text { Datum: } & \text { 16. September 2017 } \\ & 9.00-16.30 \text { Uhr } \\ \text { Ort: } & \text { Düsseldorf, Airport-Hotel Van der Valk } \\ \text { Veranstalter: } & \begin{array}{l}\text { InfectoPharm GmbH } \\ \text { Tel: 06252 95 7555, Fax: 06252 958846 }\end{array} \\ \text { Programm: } & \text { www.consilium-fortbildung.de/HNO0917 } \\ \text { Anmeldung: } & \text { www.infectopharm.com/fortbildung } \\ & \text { (für registrierte Nutzer) }\end{array}$

bzw. Verdacht auf eine Tumorerkrankung

_Zustand nach Peritonsillarabszess (bei schwergreifenden Entzündungen entstehen Narben im Gewebe, die zu Abschottungen der Krypten in diesem Bereich führen und somit weitere schwere Abszesse hervorrufen können).

Es wird immer wieder die (relative!) Indikation bei Mononukleose zur Verringerung der Viruslast diskutiert, weil dadurch der Erkrankungsverlauf verkürzt werden könnte. Das größte Problem hierbei wäre, dass man eine sogenannte "heiße TE“ durchführen müsste, die immer sehr problematisch ist, weil das hochentzündete Gewebe viel stärker blutet als bei einer Ausschälung der nicht entzündeten Mandel.

Die S2K-Leitlinie 017/024 (2015) hat klar geregelt, dass die Tonsillektomie als Routinemaßnahme zur Symptomkontrolle oder Abkürzung des Krankheitsverlaufes nicht durchgeführt werden soll. Bei Atemwegsobstruktion und drohender Ateminsuffizienz ist die Tonsillektomie indiziert. Darüber hinaus gibt es keine randomisiert-kontrollierte Studie zur Tonsillektomie bei Mononukleose zwischen 1984 und 2014! Auch moderne internationale Leitlinien zur Tonsillektomie (Schottland, Frankreich, USA) erwähnen Indikationen bei Mononukleose nicht.

\section{Literatur}

1. Dt. Ärzteblatt 13, 110 (22) A-1098

2. Laryngoscope 2011; $121: 2322-2326$

\section{Prof. Dr. med. Heidrun Müller}

Klinik und Poliklinik für Hals-NasenOhrenheilkunde/Plastische Operationen Universitätsklinikum Leipzig

Liebigstraße 18

04103 Leipzig 